Mathematical Modelling and Analysis

Volume 19 Number 3, June 2014, 395-416

http://dx.doi.org/10.3846/13926292.2014.925984

(c) Vilnius Gediminas Technical University, 2014
Publisher: Taylor\&Francis and VGTU

http://www.tandfonline.com/TMMA

Print ISSN: 1392-6292

Online ISSN: 1648-3510

\title{
Multiple Positive Solutions of BVPs for Singular Fractional Differential Equations with Non-Caratheodory Nonlinearities*
}

\section{Yuji Liu}

\author{
Guangdong University of Finance and Economics \\ Guanghou, Guangdong Province, 510320, China \\ E-mail: liuyuji888@sohu.com
}

Received June 12, 2012; revised April 26, 2014; published online June 1, 2014

\begin{abstract}
In this article, the existence of multiple positive solutions of boundaryvalue problems for nonlinear singular fractional order elastic beam equations is established. Here $f$ depends on $x, x^{\prime}$ and $x^{\prime \prime}, f$ may be singular at $t=0$ and $t=1$ and $f$ is non-Caratheodory function. The analysis relies on the well known Schauder fixed point theorem and the five functional fixed point theorems in the cones.
\end{abstract}

Keywords: positive solution, singular fractional differential equation, fixed-point theorem, fractional order elastic beam equation, non-Caratheodory function.

AMS Subject Classification: 92D25; 34A37; 34K15.

\section{Introduction}

Fourth order two-point boundary value problems are useful for material mechanics because the problems usually characterize the deflection of an elastic beam. The following problem

$$
\left\{\begin{array}{l}
u^{\prime \prime \prime \prime}(t)=f(t, u(t)), \quad t \in(0,1), \\
u(0)=u(1)=u^{\prime}(0)=u^{\prime}(1)=0
\end{array}\right.
$$

describes the deflection of an elastic beam with both ends rigidly fixed. The existence of positive solutions of (1.1) was studied extensively, see $[1,6,20,25]$. In known papers, the assumptions imposed on $f$ is continuous on $[0,1] \times R$ or is Caratheodory function.

Differential equations with fractional-order derivatives/integrals are called fractional differential equations. In some real world problems, fractional-order

* Supported by the Natural Science Foundation of Guangdong province (No. S2011010001900) and the Guangdong Higher Education Foundation for High-level talents. 
models (or fractional differential models) are found to be more adequate than integer-order models. The last two decades have witnessed a great progress in fractional calculus and fractional-order dynamical systems. It has been found that fractional calculus is a mathematical tool that works adequately for anomalous social and physical systems with nonlocal, frequency and historydependent properties, and for intermediate states such as soft materials, which are neither ideal solid nor ideal fluid (see $[3,12,13,14]$ ).

Some basic theory for the initial value problems of fractional differential equations has been discussed by Lakshmikantham [16], El-Sayed et al. [11]. Mathematical aspects of studies on fractional differential equations were discussed by many authors, see text books [21] and [23], papers [2,7,9,10,15, 22, $24,26]$ and references therein.

The use of cone theoretic techniques in studies of the existence of positive solutions of boundary value problems for differential equations with fractional order $\alpha \in(1,2]$ has a rich and diverse history [7,9,10,24,26]. Moreover, there are some works that deal with the existence and multiplicity of solutions to nonlinear fractional differential equations by using a fixed-point theorem or the topological degree theory.

In [24], the authors studied the existence of positive solutions of the following boundary value problem (a generalization of BVP (1.1)) for the fractional order beam equation

$$
\left\{\begin{array}{l}
D_{0^{+}}^{\alpha} u(t)=f(t, u(t)), \quad t \in(0,1), \\
u(0)=u(1)=u^{\prime}(0)=u^{\prime}(1)=0
\end{array}\right.
$$

where $3<\alpha \leq 4, D_{0^{+}}^{\alpha}$ ( $D^{\alpha}$ for short) is the Riemann-Liouville fractional derivative of order $\alpha$, and $f:[0,1] \times[0, \infty) \rightarrow[0, \infty)$ is continuous or $f:$ $[0,1] \times(0, \infty) \rightarrow[0, \infty)$ is continuous and $f$ is singular at $x=0$. We note that $f$ in (1.2) depends on $x, t \rightarrow f(t, x)$ is continuous on [0,1], and the solutions obtained in [24] satisfy that both $x$ and $x^{\prime}$ are continuous on $[0,1]$ (hence they are bounded on $[0,1])$. In [18], authors studied the existence of positive solutions for fractional order elastic beam equation

$$
\left\{\begin{array}{l}
D_{0^{+}}^{\alpha} u(t)=f(t, u(t)), \quad t \in(0,1), \\
u(0)=u^{\prime \prime}(0)=u^{\prime}(0)=u^{\prime \prime}(1)=0,
\end{array}\right.
$$

where $3<\alpha \leq 4, D_{0^{+}}^{\alpha}$ ( $D^{\alpha}$ for short) is the Riemann-Liouville fractional derivative of order $\alpha$, and $f:[0,1] \times[0, \infty) \rightarrow[0, \infty)$ is a continuous function. A question appears: does there exist solutions when $f$ in (1.2) or even in (1.1) is not a Caratheodory function? Let $\sigma(t)=t^{-\frac{1}{2}}(1-t)^{-\frac{3}{2}}$. It is easy to see that $\sigma \notin L^{1}(0,1) \cup C^{0}[0,1]$. Consider the following problem

$$
\left\{\begin{array}{l}
D_{0^{+}}^{\alpha} x(t)=\sigma(t), \\
\lim _{t \rightarrow 0} t^{4-\alpha} x(t)=\lim _{t \rightarrow 0} t^{4-\alpha} x^{\prime}(t)=x(1)=x^{\prime}(1)=0 .
\end{array}\right.
$$

Here, $3<\alpha<4$. Since $(1+x)^{\mu} \geq 1+\mu x$ for all $x \geq 0$ and $\mu<0$, then

$$
\int_{0}^{t} \frac{(t-s)^{\alpha-2}}{\Gamma(\alpha-1)} s^{-\frac{1}{2}}(1-s)^{-\frac{3}{2}} d s \geq(1-t)^{-\frac{3}{2}} t^{\alpha-\frac{3}{2}} \int_{0}^{1} \frac{(1-w)^{\alpha-2}}{\Gamma(\alpha-1)} w^{-\frac{1}{2}} d w
$$




$$
-\frac{3}{2}(1-t)^{-\frac{5}{2}} t^{\alpha-\frac{5}{2}} \int_{0}^{1} \frac{(1-w)^{\alpha-3}}{\Gamma(\alpha-1)} w^{-\frac{1}{2}} d w \rightarrow \infty, \quad t \rightarrow 1 .
$$

Then problem (1.4) has a unique solution

$$
\begin{aligned}
x(t)= & \int_{0}^{t} \frac{(t-s)^{\alpha-1}}{\Gamma(\alpha)} \sigma(s) d s \\
& +t^{\alpha-1}\left((\alpha-2) \int_{0}^{1} \frac{(1-s)^{\alpha-1}}{\Gamma(\alpha)} \sigma(s) d s-\int_{0}^{1} \frac{(1-s)^{\alpha-2}}{\Gamma(\alpha-1)} \sigma(s) d s\right) \\
& +t^{\alpha-2}\left(\int_{0}^{1} \frac{(1-s)^{\alpha-2}}{\Gamma(\alpha-1)} \sigma(s) d s-(\alpha-1) \int_{0}^{1} \frac{(1-s)^{\alpha-1}}{\Gamma(\alpha)} \sigma(s) d s\right)
\end{aligned}
$$

satisfying that $x$ is bounded but $x^{\prime}$ is unbounded on $(0,1]$, while $\sigma$ is not a Caratheodory function. Hence it is interesting to study the solvability of BVP (1.2) with $f$ being a non-Caratheodory function.

For nonlinear fractional order equations in (1.2) and (1.3), only the situation that the nonlinear term does not depend on the first order derivative are considered. It is interesting to deal with the situation that lower order derivatives are involved in the nonlinear term explicitly. In fact, the derivatives are of great importance in the problem in some cases. For example, in the linear elastic beam equation (Euler-Bernoulli equation)

$$
\left(E I u^{\prime \prime}(t)\right)^{\prime \prime}=f(t), \quad t \in(0, L),
$$

where $u(t)$ is the deformation function, $L$ is the length of the beam, $f(t)$ is the load density, $E$ is the Youngs modulus of elasticity and $I$ is the moment of inertia of the cross-section of the beam. In this problem, the physical meaning of the derivatives of the function $u(t)$ is as follows: $u^{(4)}(t)$ is the load density stiffness, and $u^{\prime}(t)$ is the slope and $u^{\prime \prime}(t)$ is the bending moment stiffness. If the payload depends on the slope and on the bending moment stiffness, the lower order derivatives of the unknown function are involved in the nonlinear term (the load density term) explicitly.

Authors in [8] obtained the continuous solutions on $[0,1]$ of boundary value problem

$$
\left\{\begin{array}{l}
{ }^{c} D_{0^{+}}^{\alpha} u(t)+f\left(t, u(t),{ }^{c} D_{0^{+}}^{\beta}(t)\right)=0, \quad t \in(0,1), \\
u(0)=u^{\prime \prime}(0)=u^{\prime}(0)=u(1)-u(\xi)=0,
\end{array}\right.
$$

where $3<\alpha \leq 4,{ }^{c} D_{0^{+}}$is the Caputo fractional derivative, $0<\beta \leq \alpha-1$, $\xi \in(0,1)$ and $f:[0,1] \times[0, \infty) \times R \rightarrow[0, \infty)$ is continuous or $f:[0,1] \times(0, \infty) \rightarrow$ $[0, \infty)$ is continuous. The methods used in [5] are based upon the Schauder fixed point theorem in Banach space.

Authors in [5] obtained the continuous positive solutions on $[0,1]$ of boundary value problem

$$
\left\{\begin{array}{l}
D_{0^{+}}^{\alpha} u(t)+a(t) f\left(t, u(t), u^{\prime \prime}(t)\right)=0, \quad t \in(0,1), \\
u(0)=u^{\prime \prime}(0)=u^{\prime}(0)=u^{\prime \prime}(1)=0
\end{array}\right.
$$

where $3<\alpha \leq 4, D_{0^{+}}^{\alpha}$ ( $D^{\alpha}$ for short) is the Riemann-Liouville fractional derivative of order $\alpha$, and $f:[0,1] \times[0, \infty) \times R \rightarrow[0, \infty)$ is continuous, 
$a:[0,1] \rightarrow[0, \infty)$ is continuous and there exists $0<w<1$ such that $\int_{w}^{1}\left[(1-s)^{\alpha-3}-(1-s)^{\alpha-1}\right] a(s) d s>0$. The methods used in [5] are based upon the fixed point theorem in cones in Banach space.

Suggested by [27], for constructing a suitable Banach space $X$, we replace boundary conditions $u(0)=0$ and $u^{\prime}(0)=0$ in BVP (1.2) by $\lim _{t \rightarrow 0} t^{4-\alpha} u(t)=$ $\lim _{t \rightarrow 0} t^{4-\alpha} u^{\prime}(t)=0$, which make the possible solutions be non-continuous on $[0,1]$.

Motivated by $[18,24,27]$ and above mentioned example and reasons, in this paper, we discuss the boundary value problem for nonlinear singular fractional order elastic beam equation of the form

$$
\left\{\begin{array}{l}
D_{0^{+}}^{\alpha} u(t)=f\left(t, u(t), u^{\prime}(t), u^{\prime \prime}(t)\right), \quad t \in(0,1), \\
\lim _{t \rightarrow 0} t^{4-\alpha} u(t)=\lim _{t \rightarrow 0} t^{4-\alpha} u^{\prime}(t)=0, \\
u(1)=u^{\prime}(1)=0
\end{array}\right.
$$

where $3<\alpha<4, D_{0^{+}}^{\alpha}$ ( $D^{\alpha}$ for short) is the Riemann-Liouville fractional derivative of order $\alpha$, and $f:(0,1) \times[0, \infty) \times R^{2} \rightarrow[0, \infty)$ is continuous. $f$ depends on $u, u^{\prime}$ and $u^{\prime \prime}$ and may be singular at $t=0$ and $t=1, f$ is a non-Caratheodory function.

The purpose of this paper is to establish some existence results for one and three positive solutions of BVP (1.7) by using the Schauder fixed point theorem and the five functionals fixed point theorem in the cones (see Theorems 1 and 2). The solutions obtained in this paper may be unbounded since $\lim _{t \rightarrow 0} t^{4-\alpha} x(t)=$ $\lim _{t \rightarrow 0} t^{4-\alpha} x^{\prime}(t)=0$. The methods used in this paper are different from those ones concerning existence of positive solutions of boundary value problems for integer order elastic beam equations used in [1]: contraction mapping and iterative techniques, [25]: Guo-Krasnosel'skii fixed point theorem, [6]: upper and lower solution methods, [19]: topological degree theory in order Banach space, [17]: fixed point theorem of generalized concave operators, [4]: the global bifurcation techniques.

A function $x:(0,1] \rightarrow R$ is called a solution of BVP (1.7) if $x \in C^{2}(0,1]$ and all equations in (1.7) are satisfied. $x$ is called a positive solution of BVP (1.7) if it is a solution of $\operatorname{BVP}(1.7)$ and $x(t) \geq 0$ for all $t \in(0,1]$.

The remainder of the paper is divided into two sections. In Section 2, we present some preliminary results. The main theorems and their proofs are given in Section 3.

\section{Preliminary Results}

For the convenience of the reader, we present here the necessary definitions from fixed point theory and fractional calculus theory. These definitions and results can be found in the literatures $[4,21,23]$.

Definition 1. [4] Let $X$ be a real Banach space. The nonempty convex closed subset $P$ of $X$ is called a cone in $X$ if $a x \in P$ for all $x \in P$ and $a \geq 0, x \in X$ and $-x \in X$ imply $x=0$. 
Definition 2. [4] A map $\psi: P \rightarrow[0,+\infty)$ is a nonnegative continuous concave (or convex) functional map if $\psi$ is nonnegative, continuous and satisfies $\psi(t x+(1-t) y) \geq t \psi(x)+(1-t) \psi(y)($ or $\psi(t x+(1-t) y) \leq t \psi(x)+(1-t) \psi(y))$ for all $x, y \in P$ and $t \in[0,1]$.

Definition 3. [4] An operator $T: X \rightarrow X$ is completely continuous if it is continuous and maps bounded sets into relatively compact sets.

To prove our results, we need the Five Functionals Fixed Point Theorem due to Avery [4] which is a generalization of the Leggett-Williams fixed point theorem, and the well known Schauder's fixed point theorem.

Suppose that $X$ is a real Banach space, $P$ a cone in $X$. Let $c_{1}, c_{2}, c_{3}, c_{4}, c_{5}>$ 0 be positive constants, $\alpha_{1}, \alpha_{2}$ be two nonnegative continuous concave functionals on the cone $P, \beta_{1}, \beta_{2}, \beta_{3}$ be three nonnegative continuous convex functionals on the cone $P$. Define the convex sets as follows:

$$
\begin{aligned}
P_{c_{5}} & =\left\{x \in P:\|x\|<c_{5}\right\}, \\
P\left(\beta_{1}, \alpha_{1} ; c_{2}, c_{5}\right) & =\left\{x \in P: \alpha_{1}(x) \geq c_{2}, \beta_{1}(x) \leq c_{5}\right\}, \\
P\left(\beta_{1}, \beta_{3}, \alpha_{1} ; c_{2}, c_{4}, c_{5}\right) & =\left\{x \in P: \alpha_{1}(x) \geq c_{2}, \beta_{3}(x) \leq c_{4}, \beta_{1}(x) \leq c_{5}\right\}, \\
Q\left(\beta_{1}, \beta_{2} ;, c_{1}, c_{5}\right) & =\left\{x \in P: \beta_{2}(x) \leq c_{1}, \beta_{1}(x) \leq c_{5}\right\}, \\
Q\left(\beta_{1}, \beta_{2}, \alpha_{2} ; c_{3}, c_{1}, c_{5}\right) & =\left\{x \in P: \alpha_{2}(x) \geq c_{3}, \beta_{2}(x) \leq c_{1}, \beta_{1}(x) \leq c_{5}\right\} .
\end{aligned}
$$

Lemma 1. [4] Let $X$ be a real Banach space, $P$ be a cone in $X . \alpha_{1}, \alpha_{2}$ be two nonnegative continuous concave functionals on the cone $P, \beta_{1}, \beta_{2}, \beta_{3}$ be three nonnegative continuous convex functionals on the cone $P$. Then $T$ has at least three fixed points $y_{1}, y_{2}$ and $y_{3}$ such that $\beta_{2}\left(y_{1}\right)<c_{1}, \alpha_{1}\left(y_{2}\right)>c_{2}$, $\beta_{2}\left(y_{3}\right)>c_{1}, \alpha_{1}\left(y_{3}\right)<c_{2}$, if

(i) $T: X \rightarrow X$ is a completely continuous operator;

(ii) there exists a constant $M>0$ such that $\alpha_{1}(x) \leq \beta_{2}(x),\|x\| \leq M \beta_{1}(x)$ for all $x \in P$;

(iii) there exist positive numbers $c_{1}, c_{2}, c_{3}, c_{4}, c_{5}$ with $c_{1}<c_{2}$ such that

(C1) $T \overline{P_{c_{5}}} \subset \overline{P_{c_{5}}}$;

(C2) $\left\{y \in P\left(\beta_{1}, \beta_{3}, \alpha_{1} ; c_{2}, c_{4}, c_{5}\right) \mid \alpha_{1}(x)>c_{2}\right\} \neq \emptyset$ and

$$
\alpha_{1}(T x)>c_{2} \quad \text { for every } x \in P\left(\beta_{1}, \beta_{3}, \alpha_{1} ; c_{2}, c_{4}, c_{5}\right) ;
$$

(C3) $\left\{y \in Q\left(\beta_{1}, \beta_{2}, \alpha_{2} ; c_{3}, c_{1}, c_{5}\right) \mid \beta_{2}(x)<c_{1}\right\} \neq \emptyset$ and

$$
\beta_{2}(T x)<c_{1} \quad \text { for every } x \in Q\left(\beta_{1}, \beta_{2}, \alpha_{2} ; c_{3}, c_{1}, c_{5}\right) ;
$$

(C4) $\alpha_{1}(T y)>c_{2}$ for each $y \in P\left(\beta_{1}, \alpha_{1} ; c_{2}, c_{5}\right)$ with $\beta_{3}(T y)>c_{4}$;

(C5) $\beta_{2}(T x)<c_{1}$ for each $x \in Q\left(\beta_{1}, \beta_{2} ; c_{1}, c_{5}\right)$ with $\alpha_{2}(T x)<c_{3}$.

Denote the Gamma and Beta functions, respectively, by

$$
\Gamma\left(\sigma_{1}\right)=\int_{0}^{+\infty} s^{\sigma_{1}} e^{-s} d s, \quad \mathbf{B}\left(\sigma_{2}, \sigma_{3}\right)=\int_{0}^{1}(1-s)^{\sigma_{2}-1} s^{\sigma_{3}-1} d s .
$$


Definition 4. [21] The Riemann-Liouville fractional integral of order $\alpha>0$ of a function $f:(0, \infty) \rightarrow R$ is given by

$$
I_{0+}^{\alpha} f(t)=\frac{1}{\Gamma(\alpha)} \int_{0}^{t}(t-s)^{\alpha-1} f(s) d s,
$$

provided that the right-hand side exists.

Definition 5. [21] The Riemann-Liouville fractional derivative of order $\alpha>0$ of a continuous function $f:(0, \infty) \rightarrow R$ is given by

$$
D_{0^{+}}^{\alpha} f(t)=\frac{1}{\Gamma(n-\alpha)} \frac{d^{n}}{d t^{n}} \int_{0}^{t} \frac{f(s)}{(t-s)^{\alpha-n+1}} d s,
$$

where $n<\alpha \leq n+1$, provided that the right-hand side is point-wise defined on $(0, \infty)$.

Lemma 2. [21] Let $n<\alpha \leq n+1, u \in C^{0}(0,1) \cap L^{1}(0,1)$. Then

$$
I_{0+}^{\alpha} D_{0+}^{\alpha} u(t)=u(t)+C_{1} t^{\alpha-1}+C_{2} t^{\alpha-2}+\cdots+C_{n} t^{\alpha-n},
$$

where $C_{i} \in R, i=1,2, \ldots, n$.

For our construction, we choose

$$
X=\left\{\begin{array}{c}
x(1)=0, x^{\prime}(1)=0 \\
x \in C^{0}(0,1], x^{\prime} \in C^{0}(0,1], x^{\prime \prime} \in C^{0}(0,1] \\
x:(0,1] \rightarrow R \quad \\
\text { there exist the limits } \\
\\
\lim _{t \rightarrow 0} t^{4-\alpha} x(t), \lim _{t \rightarrow 0} t^{4-\alpha} x^{\prime}(t), \\
\lim _{t \rightarrow 0} t^{4-\alpha} x^{\prime \prime}(t)
\end{array}\right\}
$$

with the norm

$$
\|u\|=\max \left\{\sup _{t \in(0,1]} t^{4-\alpha}|u(t)|, \sup _{t \in(0,1]} t^{4-\alpha}\left|u^{\prime}(t)\right|, \sup _{t \in(0,1]} t^{4-\alpha}\left|u^{\prime \prime}(t)\right|\right\}
$$

for $u \in X$. It is easy to show that $X$ is a real Banach space.

Lemma 3. Suppose that $h \in C^{0}(0,1)$ and there exist $\sigma \in(2-\alpha,-1)$ and $k \in(-2-\sigma, \alpha-4)$ such that $|h(t)| \leq t^{k}(1-t)^{\sigma}$ for all $t \in(0,1)$. Then $x \in X$ is a solution of problem

$$
\left\{\begin{array}{l}
D^{\alpha} x(t)=h(t), \quad 0<t<1, \\
\lim _{t \rightarrow 0} t^{4-\alpha} x(t)=\lim _{t \rightarrow 0} t^{4-\alpha} x^{\prime}(t)=0, \\
x(1)=x^{\prime}(1)=0
\end{array}\right.
$$

if and only if $x \in X$ satisfies

$$
x(t)=\int_{0}^{1} G(t, s) h(s) d s,
$$

where $G(t, s)$ is defined by

$$
G(t, s)=\left\{\begin{array}{l}
\frac{(t-s)^{\alpha-1}+(1-s)^{\alpha-2} t^{\alpha-2}[(s-t)+(\alpha-2)(1-t) s]}{\Gamma(\alpha)}, \quad 0 \leq s \leq t \leq 1, \\
\frac{t^{\alpha-2}(1-s)^{\alpha-2}[(s-t)+(\alpha-2)(1-t) s]}{\Gamma(\alpha)}, \quad 0 \leq t \leq s \leq 1 .
\end{array}\right.
$$


Proof. Since $h \in C^{0}(0,1)$ and there exist $\sigma \in(2-\alpha,-1)$ and $k \in(-2-\sigma$, $\alpha-4)$ such that $|h(t)| \leq t^{k}(1-t)^{\sigma}$ for all $t \in(0,1)$, then

$$
\begin{aligned}
&\left|\int_{0}^{t} \frac{(t-s)^{\alpha-1}}{\Gamma(\alpha)} s^{k}(1-s)^{\sigma} d s\right| \leq t^{\alpha+\sigma+k} \frac{\mathbf{B}(\alpha+\sigma, k+1)}{\Gamma(\alpha)}, \\
&\left|\int_{0}^{t} \frac{(t-s)^{\alpha-2}}{\Gamma(\alpha-1)} s^{k}(1-s)^{\sigma} d s\right| \leq t^{\alpha+\sigma+k-1} \frac{\mathbf{B}(\alpha+\sigma-1, k+1)}{\Gamma(\alpha-1)}, \\
&\left|\int_{0}^{t} \frac{(t-s)^{\alpha-3}}{\Gamma(\alpha-2)} s^{k}(1-s)^{\sigma} d s\right| \leq t^{\alpha+\sigma+k-2} \frac{\mathbf{B}(\alpha+\sigma-2, k+1)}{\Gamma(\alpha-2)} .
\end{aligned}
$$

So, for $t \in(0,1], D^{\alpha} u(t)=h(t)$ together with Lemma 2 implies that there exist constants $c_{i}(i=1,2,3,4)$ such that

$$
x(t)=\int_{0}^{t} \frac{(t-s)^{\alpha-1}}{\Gamma(\alpha)} h(s) d s+c_{1} t^{\alpha-1}+c_{2} t^{\alpha-2}+c_{3} t^{\alpha-3}+c_{4} t^{\alpha-4}
$$

with

$$
\begin{aligned}
x^{\prime}(t)= & \int_{0}^{t} \frac{(t-s)^{\alpha-2}}{\Gamma(\alpha-1)} h(s) d s+\frac{c_{1} \Gamma(\alpha)}{\Gamma(\alpha-1)} t^{\alpha-2} \\
& +\frac{c_{2} \Gamma(\alpha-1)}{\Gamma(\alpha-2)} t^{\alpha-3}+\frac{c_{3} \Gamma(\alpha-2)}{\Gamma(\alpha-3)} t^{\alpha-4}+\frac{\Gamma(\alpha-3)}{\Gamma(\alpha-4)} c_{4} t^{\alpha-5} \\
x^{\prime \prime}(t)= & \int_{0}^{t} \frac{(t-s)^{\alpha-3}}{\Gamma(\alpha-2)} h(s) d s+\frac{c_{1} \Gamma(\alpha)}{\Gamma(\alpha-2)} t^{\alpha-3} \\
& +\frac{c_{2} \Gamma(\alpha-1)}{\Gamma(\alpha-3)} t^{\alpha-4}+\frac{c_{3} \Gamma(\alpha-2)}{\Gamma(\alpha-4)} t^{\alpha-5}+\frac{\Gamma(\alpha-3)}{\Gamma(\alpha-5)} c_{4} t^{\alpha-6} .
\end{aligned}
$$

Now, from (2.4) $(\sigma \in(2-\alpha,-1)$ and $k \in(-2-\sigma, \alpha-4))$ and (2.7), $\lim _{t \rightarrow 0} t^{4-\alpha} x(t)=0$ implies that $c_{4}=0$. Hence from $(2.5)(\sigma \in(2-\alpha,-1)$ and $k \in(-2-\sigma, \alpha-4))$ and $(2.8), \lim _{t \rightarrow 0} t^{4-\alpha} x^{\prime}(t)=0$ implies $c_{3}=0$. Thus (2.9) with $x(1)=x^{\prime}(1)=0$ implies that

$$
\begin{aligned}
& c_{1}=(\alpha-2) \int_{0}^{1} \frac{(1-s)^{\alpha-1}}{\Gamma(\alpha)} h(s) d s-\int_{0}^{1} \frac{(1-s)^{\alpha-2}}{\Gamma(\alpha-1)} h(s) d s, \\
& c_{2}=\int_{0}^{1} \frac{(1-s)^{\alpha-2}}{\Gamma(\alpha-1)} h(s) d s-(\alpha-1) \int_{0}^{1} \frac{(1-s)^{\alpha-1}}{\Gamma(\alpha)} h(s) d s .
\end{aligned}
$$

Substituting $c_{1}, c_{2}, c_{3}$ and $c_{4}$ into (2.7), we get

$$
x(t)=\int_{0}^{1} G(t, s) h(s) d s, \quad G \text { is defined by }(2.3) .
$$

It is easy to see from $(2.4)-(2.6), \sigma \in(2-\alpha,-1), k \in(-2-\sigma, \alpha-4)$ that $x, x^{\prime}, x^{\prime \prime} \in C^{0}(0,1]$ and

$$
\lim _{t \rightarrow 0} t^{4-\alpha} x(t)=0, \quad \lim _{t \rightarrow 0} t^{4-\alpha} x^{\prime}(t)=0, \quad \lim _{t \rightarrow 0} t^{4-\alpha} x^{\prime \prime}(t)=\frac{c_{2} \Gamma(\alpha-1)}{\Gamma(\alpha-3)}
$$

and $x(1)=x^{\prime}(1)=0$. Hence $x \in X$ and satisfies (2.2).

On the other hand, if $x \in X$ satisfies (2.2), we can prove that $x$ is a solution of problem (2.1) and $x \in X$. The proof is completed. 
Lemma 4. [11, Lemma 2.4] Let $G(t, s)$ be defined by (2.3). Then

$$
\begin{aligned}
& G(t, s) \leq \frac{\max \left\{\alpha-1,(\alpha-2)^{2}\right\} s^{2}(1-s)^{\alpha-2}}{\Gamma(\alpha)}, \quad t, s \in[0,1], \\
& G(t, s) \geq \frac{(\alpha-2) t^{\alpha-2}(1-t)^{2} s^{2}(1-s)^{\alpha-2}}{\Gamma(\alpha)}, \quad t, s \in[0,1] .
\end{aligned}
$$

Lemma 5. Let $0<p<\frac{1}{2}<q<1$. Suppose that $h \in C^{0}(0,1)$ is nonnegative and there exist $\sigma \in(2-\alpha,-1)$ and $k \in(-2-\sigma, \alpha-4)$ such that $|h(t)| \leq t^{k}(1-t)^{\sigma}$ for all $t \in(0,1)$. Then the unique solution of $B V P(2.1)$ satisfies

$$
\min _{t \in[p, q]} t^{4-\alpha} u(t) \geq \mu \sup _{t \in(0,1]} t^{4-\alpha} u(t)
$$

where $\mu$ is defined by

$$
\mu=\frac{(\alpha-2) \min \left\{p^{2}(1-p)^{2}, q^{2}(1-q)^{2}\right\}}{\max \left\{\alpha-1,(\alpha-2)^{2}\right\}} .
$$

Proof. Suppose that $u$ is a solution of BVP (2.1). By Lemmas 3 and 4, we have

$$
u(t)=\int_{0}^{1} G(t, s) h(s) d s \geq 0, \quad t \in(0,1] .
$$

Since $\left[t^{2}(1-t)^{2}\right]^{\prime}=2 t(1-t)(1-2 t)$ and $0<p<\frac{1}{2}<q<1$, we have from (2.11) that

$$
\min _{t \in[p, q]} t^{4-\alpha} G(t, s) \geq \frac{(\alpha-2) \min \left\{p^{2}(1-p)^{2}, q^{2}(1-q)^{2}\right\}}{\Gamma(\alpha)} s^{2}(1-s)^{\alpha-2}, s \in[0,1] .
$$

It follows from (2.10) that

$$
\min _{t \in[p, q]} t^{4-\alpha} u(t) \geq \mu \int_{0}^{1} G(t, s) h(s) d s=\mu t^{4-\alpha} u(t) .
$$

Then

$$
\min _{t \in[p, q]} t^{4-\alpha} u(t) \geq \mu \sup _{t \in(0,1]} t^{4-\alpha} u(t) .
$$

The proof is completed.

We seek solutions of BVP (1.7) that lie in the cone

$$
P=\left\{\begin{array}{c}
u(t) \geq 0, \quad t \in(0,1] \\
u \in X: \quad(1)=0, \quad u^{\prime}(1)=0, \\
\min _{t \in[p, q]} t^{4-\alpha} u(t) \geq \mu \sup _{t \in(0,1]} t^{4-\alpha} u(t)
\end{array}\right\} .
$$

Define the operator $T$ on $P$ by

$$
(T u)(t)=\int_{0}^{1} G(t, s) f\left(s, u(s), u^{\prime}(s), u^{\prime \prime}(s)\right) d s .
$$

By Lemma 3, we have that $x \in P$ is a positive solution if and only if $x \in P$ is a fixed point of $T$. 
Lemma 6. Suppose that $f(t, x, y, z)$ is continuous on $(0,1) \times[0, \infty) \times R^{2}$ and satisfies that for each $r>0$ there exist $\sigma \in(2-\alpha,-1)$ and $k \in(-2-\sigma, \alpha-4)$ and $M_{r}>0$ such that

$$
\left|f\left(t, t^{\alpha-4} x, t^{\alpha-4} y, t^{\alpha-4} z\right)\right| \leq M_{r} t^{k}(1-t)^{\sigma} \quad \text { for all } t \in(0,1),|x|,|y|,|z| \leq r .
$$

Then $T: P \rightarrow P$ is completely continuous.

Proof. We divide the proof into four steps.

Step 1. We prove that $T: P \rightarrow P$ is well defined.

For $x \in P$, we find $x(t) \geq 0$ for all $t \in[0,1]$ and there exits $r>0$ such that

$$
\|x\|=\max \left\{\sup _{t \in(0,1]} t^{4-\alpha}|x(t)|, \sup _{t \in(0,1]} t^{4-\alpha}\left|x^{\prime}(t)\right|, \sup _{t \in(0,1]} t^{4-\alpha}\left|x^{\prime \prime}(t)\right|\right\} \leq r .
$$

Then there exist $\sigma \in(2-\alpha,-1)$ and $k \in(-2-\sigma, \alpha-4), M_{r} \geq 0$ such that

$$
f\left(t, x(t), x^{\prime}(t), x^{\prime \prime}(t)\right) \leq M_{r} t^{k}(1-t)^{\sigma}
$$

for all $t \in(0,1)$. Since $f$ is nonnegative, and Lemma 4 , we get

$$
(T x)(t)=\int_{0}^{1} G(t, s) f\left(s, x(s), x^{\prime}(s), x^{\prime \prime}(s)\right) d s \geq 0, \quad t \in(0,1) .
$$

From (2.13) and that $f$ is nonnegative, similarly to the proof of Lemma 3, we can show that $T x \in X$.

By the same methods used in Lemma 5 , together with that $f$ is nonnegative, we can prove that

$$
\min _{t \in[p, q]} t^{4-\alpha}(T x)(t) \geq \mu \sup _{t \in(0,1]} t^{4-\alpha}(T x)(t) .
$$

So $T x \in P$. So $T: P \rightarrow P$ is well defined.

Step 2. $T$ is continuous.

Let $\left\{x_{n} \in P\right\}$ be a sequence such that $x_{n} \rightarrow x_{0}$ as $n \rightarrow \infty$ in $X$. Then there exists $r>0$ such that

$$
\max \left\{\sup _{t \in(0,1]} t^{4-\alpha}\left|x_{n}(t)\right|, \sup _{t \in(0,1]} t^{4-\alpha}\left|x_{n}^{\prime}(t)\right|, \sup _{t \in(0,1]} t^{4-\alpha}\left|x_{n}^{\prime \prime}(t)\right|\right\} \leq r
$$

for $n=0,1,2, \ldots$ Then similarly to $(2.13)$ there exists $M_{r}>0$ such that

$$
\left|f\left(t, x_{n}(t), x_{n}^{\prime}(t), x_{n}^{\prime \prime}(t)\right)\right| \leq M_{r} t^{k}(1-t)^{\sigma}
$$

holds for all $t \in(0,1), n=0,1,2, \ldots$ Then

$$
\begin{aligned}
& t^{4-\alpha}\left|\left(T x_{n}\right)(t)-\left(T x_{0}\right)(t)\right| \leq 2 M_{r} \\
& \quad \times\left[\frac{\mathbf{B}(\alpha+\sigma, k+1)}{\Gamma(\alpha)}+(2 \alpha-3) \frac{\mathbf{B}(\alpha+\sigma, k+1)}{\Gamma(\alpha)}+2 \frac{\mathbf{B}(\alpha+\sigma-1, k+1)}{\Gamma(\alpha-1)}\right]
\end{aligned}
$$


and similarly we get

$$
\begin{aligned}
t^{4-\alpha} \mid\left(T x_{n}\right)^{\prime}(t) & -\left(T x_{0}\right)^{\prime}(t) \mid \leq 2 M_{r}\left[\frac{\mathbf{B}(\alpha+\sigma-1, k+1)}{\Gamma(\alpha-1)}\right. \\
& +\left((\alpha-2) \frac{\Gamma(\alpha)}{\Gamma(\alpha-1)}+(\alpha-1) \frac{\Gamma(\alpha-1)}{\Gamma(\alpha-2)}\right) \frac{\mathbf{B}(\alpha+\sigma, k+1)}{\Gamma(\alpha)} \\
& \left.+\left(\frac{\Gamma(\alpha-1)}{\Gamma(\alpha-2)}+\frac{\Gamma(\alpha)}{\Gamma(\alpha-1)}\right) \frac{\mathbf{B}(\alpha+\sigma-1, k+1)}{\Gamma(\alpha-1)}\right]
\end{aligned}
$$

and

$$
\begin{aligned}
t^{4-\alpha} \mid\left(T x_{n}\right)^{\prime \prime}(t) & -\left(T x_{0}\right)^{\prime \prime}(t) \mid \leq 2 M_{r}\left[\frac{\mathbf{B}(\alpha+\sigma-2, k+1)}{\Gamma(\alpha-2)}\right. \\
& +\left((\alpha-2) \frac{\Gamma(\alpha)}{\Gamma(\alpha-2)}+(\alpha-1) \frac{\Gamma(\alpha-1)}{\Gamma(\alpha-3)}\right) \frac{\mathbf{B}(\alpha+\sigma, k+1)}{\Gamma(\alpha)} \\
& \left.+\left(\frac{\Gamma(\alpha-1)}{\Gamma(\alpha-3)}+\frac{\Gamma(\alpha)}{\Gamma(\alpha-2)}\right) \frac{\mathbf{B}(\alpha+\sigma-1, k+1)}{\Gamma(\alpha-1)}\right] .
\end{aligned}
$$

Since $f(t, x, y, z)$ is continuous in $x, y, z$, by dominant convergence theorem, we have $\left\|T x_{n}-T x_{0}\right\| \rightarrow 0$ as $n \rightarrow \infty$. Then $T$ is continuous.

Let $\Omega \subset P$ be a bounded subset. Then there exists $r>0$ such that

$\max \left\{\sup _{t \in(0,1]} t^{4-\alpha}|x(t)|, \sup _{t \in(0,1]} t^{4-\alpha}\left|x^{\prime}(t)\right|, \sup _{t \in(0,1]} t^{4-\alpha}\left|x^{\prime \prime}(t)\right|\right\} \leq r, \quad x \in \Omega$.

Then there exists $M_{r}>0$ such that (2.13) holds for all $t \in(0,1), x \in \Omega$.

Step 3. Prove that $T \Omega$ is a bounded set in $X$.

$$
\begin{aligned}
t^{4-\alpha}|(T x)(t)| \leq M_{r} & {\left[\frac{\mathbf{B}(\alpha+\sigma, k+1)}{\Gamma(\alpha)}+(2 \alpha-3) \frac{\mathbf{B}(\alpha+\sigma, k+1)}{\Gamma(\alpha)}\right.} \\
+ & \left.2 \frac{\mathbf{B}(\alpha+\sigma-1, k+1)}{\Gamma(\alpha-1)}\right]
\end{aligned}
$$

and similarly we get

$$
\begin{aligned}
t^{4-\alpha}\left|(T x)^{\prime}(t)\right| \leq & M_{r}\left[\frac{\mathbf{B}(\alpha+\sigma-1, k+1)}{\Gamma(\alpha-1)}\right. \\
& +\left((\alpha-2) \frac{\Gamma(\alpha)}{\Gamma(\alpha-1)}+(\alpha-1) \frac{\Gamma(\alpha-1)}{\Gamma(\alpha-2)}\right) \frac{\mathbf{B}(\alpha+\sigma, k+1)}{\Gamma(\alpha)} \\
& \left.+\left(\frac{\Gamma(\alpha-1)}{\Gamma(\alpha-2)}+\frac{\Gamma(\alpha)}{\Gamma(\alpha-1)}\right) \frac{\mathbf{B}(\alpha+\sigma-1, k+1)}{\Gamma(\alpha-1)}\right]
\end{aligned}
$$

and

$$
\begin{aligned}
t^{4-\alpha}\left|(T x)^{\prime \prime}(t)\right| \leq & M_{r}\left[\frac{\mathbf{B}(\alpha+\sigma-2, k+1)}{\Gamma(\alpha-2)}\right. \\
& +\left((\alpha-2) \frac{\Gamma(\alpha)}{\Gamma(\alpha-2)}+(\alpha-1) \frac{\Gamma(\alpha-1)}{\Gamma(\alpha-3)}\right) \frac{\mathbf{B}(\alpha+\sigma, k+1)}{\Gamma(\alpha)} \\
& \left.+\left(\frac{\Gamma(\alpha-1)}{\Gamma(\alpha-3)}+\frac{\Gamma(\alpha)}{\Gamma(\alpha-2)}\right) \frac{\mathbf{B}(\alpha+\sigma-1, k+1)}{\Gamma(\alpha-1)}\right] .
\end{aligned}
$$

So $T$ maps bounded sets into bounded sets in $X$. 
Step 4. Prove that $T \Omega$ is a relatively compact set in $X$.

We prove that both $\{T u: u \in \Omega\}$ and $\left\{(T u)^{\prime}: u \in \Omega\right\}$ are equi-continuous on $(0,1]$, and $\left\{(T u)^{\prime \prime}: u \in \Omega\right\}$ is equi-continuous on each $[e, h] \subset(0,1]$ and is equi-convergent at $t=0$. Firstly, let $t_{1}, t_{2} \in[0,1]$ with $t_{1}<t_{2}$ and $x \in \Omega$. Then we have

$$
\begin{aligned}
\mid t_{1}^{4-\alpha}(T x)\left(t_{1}\right) & -t_{2}^{4-\alpha}(T x)\left(t_{2}\right)|\leq| t_{1}^{4-\alpha} \int_{0}^{t_{1}} \frac{\left(t_{1}-s\right)^{\alpha-1}}{\Gamma(\alpha)} f\left(s, x(s), x^{\prime}(s), x^{\prime \prime}(s)\right) d s \\
& -t_{2}^{4-\alpha} \int_{0}^{t_{2}} \frac{\left(t_{2}-s\right)^{\alpha-1}}{\Gamma(\alpha)} f\left(s, x(s), x^{\prime}(s), x^{\prime \prime}(s)\right) d s \mid \\
& +M_{r}\left|(\alpha-2)\left[t_{2}^{3}-t_{1}^{3}\right]+(\alpha-1)\left[t_{1}^{2}-t_{2}^{2}\right]\right| \frac{\mathbf{B}(\alpha+\sigma, k+1)}{\Gamma(\alpha)} \\
& +M_{r}\left|t_{1}^{2}-t_{2}^{2}+t_{2}^{3}-t_{1}^{3}\right| \frac{\mathbf{B}(\alpha+\sigma-1, k+1)}{\Gamma(\alpha-1)} s^{k}(1-s)^{\sigma} d s .
\end{aligned}
$$

Since

$$
\begin{aligned}
& \mid t_{1}^{4-\alpha} \int_{0}^{t_{1}} \frac{\left(t_{1}-s\right)^{\alpha-1}}{\Gamma(\alpha)} f\left(s, x(s), x^{\prime}(s), x^{\prime \prime}(s)\right) d s \\
& \quad-t_{2}^{4-\alpha} \int_{0}^{t_{2}} \frac{\left(t_{2}-s\right)^{\alpha-1}}{\Gamma(\alpha)} f\left(s, x(s), x^{\prime}(s), x^{\prime \prime}(s)\right) d s \mid \\
& \leq M_{r}\left|t_{2}^{4-\alpha}-t_{1}^{4-\alpha}\right| \int_{0}^{t_{2}} \frac{\left(t_{2}-s\right)^{\alpha-1}}{\Gamma(\alpha)} s^{k}(1-s)^{\sigma} d s \\
& \quad+M_{r} t_{1}^{4-\alpha} \int_{t_{1}}^{t_{2}} \frac{\left(t_{2}-s\right)^{\alpha-1}}{\Gamma(\alpha)} s^{k}(1-s)^{\sigma} d s \\
& \quad+M_{r} t_{1}^{4-\alpha} \int_{0}^{t_{1}} \frac{\left|\left(t_{1}-s\right)^{\alpha-1}-\left(t_{2}-s\right)^{\alpha-1}\right|}{\Gamma(\alpha)} s^{k}(1-s)^{\sigma} d s .
\end{aligned}
$$

We can prove that $1-x^{\tau} \leq \frac{\tau}{\tau-2}(1-x)^{\tau-2}$ for all $x \in[0,1]$ and $\tau \in(2,3)$.

Hence

$$
\begin{aligned}
\mid t_{1}^{4-\alpha} & \int_{0}^{t_{1}} \frac{\left(t_{1}-s\right)^{\alpha-1}}{\Gamma(\alpha)} f\left(s, x(s), x^{\prime}(s), x^{\prime \prime}(s)\right) d s \\
& -t_{2}^{4-\alpha} \int_{0}^{t_{2}} \frac{\left(t_{2}-s\right)^{\alpha-1}}{\Gamma(\alpha)} f\left(s, x(s), x^{\prime}(s), x^{\prime \prime}(s)\right) d s \mid \\
\leq & M_{r}\left|t_{2}^{4-\alpha}-t_{1}^{4-\alpha}\right| \int_{0}^{1} \frac{(1-w)^{\alpha+\sigma-1}}{\Gamma(\alpha)} w^{k} d w+M_{r} \int_{t_{1} / t_{2}}^{1} \frac{(1-w)^{\alpha+\sigma-1}}{\Gamma(\alpha)} w^{k} d w \\
& +M_{r} \frac{\alpha-1}{\alpha-3} \int_{0}^{t_{2}} \frac{\left(t_{2}-s\right)^{2}\left(t_{2}-t_{1}\right)^{\alpha-3}}{\Gamma(\alpha)} s^{k}\left(t_{2}-s\right)^{\sigma} d s \\
\leq & M_{r}\left|t_{2}^{4-\alpha}-t_{1}^{4-\alpha}\right| \int_{0}^{1} \frac{(1-w)^{\alpha+\sigma-1}}{\Gamma(\alpha)} w^{k} d w \\
& +M_{r} \int_{t_{1} / t_{2}}^{1} \frac{(1-w)^{\alpha+\sigma-1}}{\Gamma(\alpha)} w^{k} d w+M_{r} \frac{\alpha-1}{\alpha-3}\left(t_{2}-t_{1}\right)^{\alpha-3} \frac{\mathbf{B}(\sigma+3, k+1)}{\Gamma(\alpha)} .
\end{aligned}
$$


As $t_{1} \rightarrow t_{2}$, the right-hand side of the above inequality tends to zero uniformly. So

$$
\left|t_{1}^{4-\alpha}(T x)\left(t_{1}\right)-t_{2}^{4-\alpha}(T x)\left(t_{2}\right)\right| \rightarrow 0 \quad \text { uniformly as } t_{1} \rightarrow t_{2} .
$$

Secondly, for $t_{1}, t_{2} \in[0,1]$ with $t_{1}<t_{2}$ and $x \in \Omega$, we get similarly that

$$
\begin{aligned}
& \left|t_{1}^{4-\alpha}(T x)^{\prime}\left(t_{1}\right)-t_{2}^{4-\alpha}(T x)^{\prime}\left(t_{2}\right)\right| \\
& \leq \mid t_{1}^{4-\alpha} \int_{0}^{t_{1}} \frac{\left(t_{1}-s\right)^{\alpha-2}}{\Gamma(\alpha-1)} f\left(s, x(s), x^{\prime}(s), x^{\prime \prime}(s)\right) d s \\
& \quad-t_{2}^{4-\alpha} \int_{0}^{t_{2}} \frac{\left(t_{2}-s\right)^{\alpha-2}}{\Gamma(\alpha-1)} f\left(s, x(s), x^{\prime}(s), x^{\prime \prime}(s)\right) d s \mid \\
& \quad+M_{r}\left|(\alpha-2) \frac{\Gamma(\alpha)}{\Gamma(\alpha-1)}\left[t_{2}^{2}-t_{1}^{2}\right]+(\alpha-1) \frac{\Gamma(\alpha-1)}{\Gamma(\alpha-2)}\left[t_{1}-t_{2}\right]\right| \\
& \quad \times \frac{\mathbf{B}(\alpha+\sigma, k+1)}{\Gamma(\alpha)}+M_{r}\left|\frac{\Gamma(\alpha-1)}{\Gamma(\alpha-2)}\left[t_{1}-t_{2}\right]+\frac{\Gamma(\alpha)}{\Gamma(\alpha-1)}\left[t_{2}^{2}-t_{1}^{2}\right]\right| \\
& \quad \times \frac{\mathbf{B}(\alpha+\sigma-1, k+1)}{\Gamma(\alpha-1)} s^{k}(1-s)^{\sigma} d s .
\end{aligned}
$$

Then

We can prove that $1-x^{\tau} \leq \frac{\tau}{\tau-2}(1-x)^{\tau-1}$ for all $x \in[0,1]$ and $\tau \in(1,2)$.

$$
a^{\alpha-2}-b^{\alpha-2} \leq \frac{\alpha-2}{\alpha-3} a(a-b)^{\alpha-3}, \quad a \geq b .
$$

Hence

$$
\begin{aligned}
& \mid t_{1}^{4-\alpha} \int_{0}^{t_{1}} \frac{\left(t_{1}-s\right)^{\alpha-2}}{\Gamma(\alpha-1)} f\left(s, x(s), x^{\prime}(s), x^{\prime \prime}(s)\right) d s \\
& \quad-t_{2}^{4-\alpha} \int_{0}^{t_{2}} \frac{\left(t_{2}-s\right)^{\alpha-2}}{\Gamma(\alpha-1)} f\left(s, x(s), x^{\prime}(s), x^{\prime \prime}(s)\right) d s \mid \\
& \leq M_{r}\left|t_{2}^{4-\alpha}-t_{1}^{4-\alpha}\right| \int_{0}^{1} \frac{(1-w)^{\alpha+\sigma-2}}{\Gamma(\alpha-1)} w^{k} d w \\
& \quad+M_{r} \int_{\frac{t_{1}}{t_{2}}}^{1} \frac{(1-w)^{\alpha+\sigma-2}}{\Gamma(\alpha-1)} w^{k} d w+M_{r} \frac{\alpha-2}{\alpha-3}\left(t_{2}-t_{1}\right)^{\alpha-3} \frac{\mathbf{B}(\sigma+2, k+1)}{\Gamma(\alpha-1)}
\end{aligned}
$$

As $t_{1} \rightarrow t_{2}$, the right-hand side of the above inequality tends to zero uniformly. So

$$
\left|t_{1}^{4-\alpha}(T x)^{\prime}\left(t_{1}\right)-t_{2}^{4-\alpha}(T x)^{\prime}\left(t_{2}\right)\right| \rightarrow 0 \quad \text { uniformly as } t_{1} \rightarrow t_{2} .
$$

Thirdly, for $t_{1}, t_{2} \in[e, h] \subset(0,1]$ with $t_{1}<t_{2}$ and $x \in \Omega$, we have

$$
\begin{aligned}
& \left|t_{1}^{4-\alpha}(T x)^{\prime \prime}\left(t_{1}\right)-t_{2}^{4-\alpha}(T x)^{\prime \prime}\left(t_{2}\right)\right| \\
& \quad \leq \mid t_{1}^{4-\alpha} \int_{0}^{t_{1}} \frac{\left(t_{1}-s\right)^{\alpha-3}}{\Gamma(\alpha-2)} f\left(s, x(s), x^{\prime}(s), x^{\prime \prime}(s)\right) d s
\end{aligned}
$$




$$
\begin{aligned}
& -t_{2}^{4-\alpha} \int_{0}^{t_{2}} \frac{\left(t_{2}-s\right)^{\alpha-3}}{\Gamma(\alpha-2)} f\left(s, x(s), x^{\prime}(s), x^{\prime \prime}(s)\right) d s \mid \\
& +M_{r}\left|(\alpha-2) \frac{\Gamma(\alpha)}{\Gamma(\alpha-2)}\left[t_{2}-t_{1}\right]\right| \frac{\mathbf{B}(\alpha+\sigma, k+1)}{\Gamma(\alpha)} \\
& +M_{r}\left|t_{1}-t_{2}\right| \frac{\Gamma(\alpha)}{\Gamma(\alpha-2)} \frac{\mathbf{B}(\alpha+\sigma-1, k+1)}{\Gamma(\alpha-1)} s^{k}(1-s)^{\sigma} d s .
\end{aligned}
$$

From $2-\alpha<\sigma<-1$, we can choose $\sigma_{0} \in(2-\alpha, \sigma)$. Then we have $1-x^{\varrho} \leq(1-x)^{\sigma_{0}+1+\varrho}$ for all $x \in[0,1]$ and $\varrho \in\left(-1-\sigma_{0}, 1\right)$.

In fact, we have $\sigma_{0}<\sigma<-1$. Then $\varrho \in\left(-1-\sigma_{0}, 1\right)$ implies $\varrho>0$ and

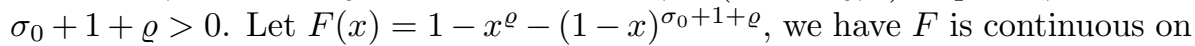
$[0,1], F(0)=0$ and $F(1)=0$. On the other hand, we have

$$
\begin{aligned}
& F^{\prime}(x)=-\varrho x^{\varrho-1}+\left(\sigma_{0}+1+\varrho\right)(1-x)^{\sigma_{0}+\varrho} \\
& F^{\prime \prime}(x)=-\varrho(\varrho-1) x^{\varrho-2}-\left(\sigma_{0}+1+\varrho\right)\left(\sigma_{0}+\varrho\right)(1-x)^{\sigma_{0}+\varrho-1}
\end{aligned}
$$

Since $\sigma_{0} \in(2-\alpha, \sigma)$ and $\varrho \in\left(-1-\sigma_{0}, 1\right)$, we know that $\varrho>0, \varrho-1<0$ and $\sigma_{0}+\varrho<0$. Then $F^{\prime \prime}(x) \geq 0$ for all $x \in(0,1)$. Hence $F(x) \leq 0$ for all $x \in[0,1]$. Then $1-x^{\varrho} \leq(1-x)^{\sigma_{0}+1+\varrho}$ for all $x \in[0,1]$ and $\varrho \in(-1-\sigma, 1)$. So

$$
1-x^{\alpha-3} \leq(1-x)^{\sigma_{0}+\alpha-2} \text { for all } x \in[0,1] \text {. }
$$

It follows that

$$
a^{\alpha-3}-b^{\alpha-3} \leq a^{-1-\sigma_{0}}(a-b)^{\sigma_{0}+\alpha-2}, \quad a \geq b \geq 0 .
$$

Hence for $[e, h] \subset(0,1]$ and $t_{1}<t_{2}$ with $t_{1}, t_{2} \in[e, h]$, we have

$$
\begin{aligned}
& \mid t_{1}^{4-\alpha} \int_{0}^{t_{1}} \frac{\left(t_{1}-s\right)^{\alpha-3}}{\Gamma(\alpha-2)} f\left(s, x(s), x^{\prime}(s), x^{\prime \prime}(s)\right) d s \\
& \quad-t_{2}^{4-\alpha} \int_{0}^{t_{2}} \frac{\left(t_{2}-s\right)^{\alpha-3}}{\Gamma(\alpha-2)} f\left(s, x(s), x^{\prime}(s), x^{\prime \prime}(s)\right) d s \mid \\
& \leq M_{r}\left|t_{2}^{4-\alpha}-t_{1}^{4-\alpha}\right| \int_{0}^{1} \frac{(1-w)^{\alpha+\sigma-3}}{\Gamma(\alpha-2)} w^{k} d w+M_{r} \int_{t_{1} / t_{2}}^{1} \frac{(1-w)^{\alpha+\sigma-3}}{\Gamma(\alpha-2)} w^{k} d w \\
& \quad+M_{r}\left[t_{2}-t_{1}\right]^{\sigma_{0}+\alpha-2} \max \left\{e^{\sigma-\sigma_{0}+k}, h^{\sigma-\sigma_{0}+k}\right\} \int_{0}^{t_{1} / t_{2}} \frac{(1-w)^{\sigma-\sigma_{0}-1}}{\Gamma(\alpha-2)} w^{k} d w .
\end{aligned}
$$

As $t_{1} \rightarrow t_{2}$, the right-hand side of the above inequality tends to zero uniformly. So

$$
\left|t_{1}^{4-\alpha}(T x)^{\prime \prime}\left(t_{1}\right)-t_{2}^{4-\alpha}(T x)^{\prime \prime}\left(t_{2}\right)\right| \rightarrow 0 \text { uniformly as } t_{1} \rightarrow t_{2} \text { on }[e, h] \subseteq(0,1] .
$$

Fourthly we have

$$
\mid t^{4-\alpha}(T x)^{\prime \prime}(t)-\left[(\alpha-1) \frac{\Gamma(\alpha-1)}{\Gamma(\alpha-3)} \int_{0}^{1} \frac{(1-s)^{\alpha-1}}{\Gamma(\alpha)}\left|f\left(s, x(s), x^{\prime}(s), x^{\prime \prime}(s)\right)\right| d s\right.
$$




$$
\begin{aligned}
& \left.+\frac{\Gamma(\alpha-1)}{\Gamma(\alpha-3)} \int_{0}^{1} \frac{(1-s)^{\alpha-2}}{\Gamma(\alpha-1)}\left|f\left(s, x(s), x^{\prime}(s), x^{\prime \prime}(s)\right)\right| d s\right] \mid \\
\leq & M_{r} t^{2+\sigma} \int_{0}^{1} \frac{(1-w)^{\alpha+\sigma-3}}{\Gamma(\alpha-2)} w^{k} d w+M_{r} t\left[(\alpha-2) \frac{\Gamma(\alpha)}{\Gamma(\alpha-2)} \frac{\mathbf{B}(\alpha+\sigma, k+1)}{\Gamma(\alpha)}\right. \\
& \left.+\frac{\Gamma(\alpha)}{\Gamma(\alpha-2)} \frac{\mathbf{B}(\alpha+\sigma-1, k+1)}{\Gamma(\alpha-1)}\right] .
\end{aligned}
$$

It follows that

$$
t^{4-\alpha}(T x)^{\prime \prime}(t) \text { is uniformly convergent as } t \rightarrow 0 .
$$

From (2.17) and (2.18), we get

$$
\left|t_{1}^{4-\alpha}(T x)^{\prime \prime}\left(t_{1}\right)-t_{2}^{4-\alpha}(T x)^{\prime \prime}\left(t_{2}\right)\right| \rightarrow 0 \quad \text { uniformly as } t_{1} \rightarrow t_{2} \text { on }(0,1] .
$$

Therefore, (2.15), (2.16) and (2.19) imply that $T \Omega$ is relatively compact. From above discussion, $T$ is completely continuous.

Remark 1. The proof in Step 4 can be simplified. Since $T: X \rightarrow P$, we can take

$$
\begin{aligned}
& \left.t^{4-\alpha} T x(t)\right|_{t=0}=\lim _{t \rightarrow 0} t^{4-\alpha}(T x)(t),\left.\quad t^{4-\alpha}(T x)^{\prime}(t)\right|_{t=0}=\lim _{t \rightarrow 0} t^{4-\alpha}(T x)^{\prime}(t), \\
& \left.t^{4-\alpha}(T x)^{\prime \prime}(t)\right|_{t=0}=\lim _{t \rightarrow 0} t^{4-\alpha}(T x)^{\prime \prime}(t) .
\end{aligned}
$$

Then $t^{4-\alpha}(T x)(t), t^{4-\alpha}(T x)^{\prime}(t)$ and $t^{4-\alpha}(T x)^{\prime \prime}(t) \in C[0,1]$ for each $x \in \Omega$. Because $t^{4-\alpha}(T x)(t)=\int_{0}^{1} t^{4-\alpha} G(t, s) f\left(s, x(s), x^{\prime}(s), x^{\prime \prime}(s)\right) d s$, and $t^{4-\alpha} G(t, s)$ is uniformly continuous, for any $\varepsilon>0$, there exists $\delta_{0}>0$, when $t_{1}, t_{2} \in[0,1]$, $\left|t_{1}-t_{2}\right|<\delta_{0}$ and $x \in \Omega$, we can get $\left|t^{4-\alpha} T x\left(t_{1}\right)-t^{4-\alpha} T x\left(t_{2}\right)\right|<\varepsilon$. Similar methods should be applied to the remaining two formulas. We omit the details.

\section{Main Results}

In this section, we prove the main results. Choose $0<p<\frac{1}{2}<q<1$. Denote

$$
\mu=\frac{(\alpha-2) \min \left\{p^{2}(1-p)^{2}, q^{2}(1-q)^{2}\right\}}{\max \left\{\alpha-1,(\alpha-2)^{2}\right\}} .
$$

Denote the following assumption by

(B0) $f(t, x, y, z)$ is continuous on $(0,1) \times[0, \infty) \times R^{2}$ and there exist $\sigma \in$ $(2-\alpha,-1)$ and $k \in(\alpha-4,-\sigma-2)$ such that for each $r>0$ there exists $M_{r}>0$ such that

$$
\left|f\left(t, t^{\alpha-4} x, t^{\alpha-4} y, t^{\alpha-4} z\right)\right| \leq M_{r} t^{k}(1-t)^{\sigma} \quad \text { for all } t \in(0,1),|x|,|y| \leq r .
$$

Theorem 1. Suppose that

(B1) $\phi:(0,1) \rightarrow R$ satisfies that there exist $\sigma_{0} \in(2-\alpha,-1)$ and $k_{0} \in(-2-$ $\left.\sigma_{0}, \alpha-4\right)$ and $M_{0}>0$ such that $|\phi(t)| \leq M_{0} t^{k_{0}}(1-t)^{\sigma_{0}}$ for all $t \in(0,1)$; 
(B2) there exist numbers $\sigma_{i} \in(2-\alpha,-1)$ and $k_{i} \in\left(-2-\sigma_{i}, \alpha-4\right)(i=$ $1,2, \ldots, m), \tau_{i 1}, \tau_{i 2}, \tau_{i 3} \geq 0(i=1,2, \ldots, m), A_{i} \geq 0(i=1,2, \ldots, m)$ such that

$$
\left|f\left(t, t^{\alpha-4} x, t^{\alpha-4} y, t^{\alpha-4} z\right)-\phi(t)\right| \leq \sum_{i=1}^{m} A_{i} t^{k_{i}}(1-t)^{\sigma_{i}}|x|^{\tau_{i 1}}|y|^{\tau_{i 2}}|z|^{\tau_{i 3}}
$$

holds for all $t \in(0,1), x, y, z \in R$.

Let $\mu_{i}=\tau_{i 1}+\tau_{i 2}+\tau_{i 3}(i=1,2, \ldots, m)$ and

$$
\begin{aligned}
P_{i}= & \frac{\mathbf{B}\left(\alpha+\sigma_{i}-2, k_{i}+1\right)}{\Gamma(\alpha-2)}+\left[(\alpha-2) \frac{\Gamma(\alpha)}{\Gamma(\alpha-2)}+(\alpha-1) \frac{\Gamma(\alpha-1)}{\Gamma(\alpha-3)}\right] \\
& \times \frac{\mathbf{B}\left(\alpha+\sigma_{i}, k_{i}+1\right)}{\Gamma(\alpha)}+\left[\frac{\Gamma(\alpha-1)}{\Gamma(\alpha-3)}+\frac{\Gamma(\alpha)}{\Gamma(\alpha-2)}\right] \frac{\mathbf{B}\left(\alpha+\sigma_{i}-1, k_{i}+1\right)}{\Gamma(\alpha-1)} .
\end{aligned}
$$

Then BVP (1.7) has at least one positive solution if

(i) $\mu=\max \left\{\mu_{i}(i=1,2, \ldots, m)\right\}<1$ or

(ii) $\mu=\max \left\{\mu_{i}(i=1,2, \ldots, m)\right\}=1$ with $\sum_{i=1}^{m} A_{i} P_{i}\|\Phi\|^{\mu_{i}-1}<1$ or

(iii) $\mu=\max \left\{\mu_{i}(i=1,2, \ldots, m)\right\}>1$ with

$$
\frac{\|\Phi\|(\mu-1)^{\mu-1}}{(\|\Phi\| \mu)^{\mu}} \geq \sum_{i=1}^{m} A_{i} P_{i}\|\Phi\|^{\mu_{i}-\mu} .
$$

Proof. It is easy to show that (B1) and (B2) imply (B0) with $k=\min \left\{k_{i}(i=\right.$ $0,1,2, \ldots, m)\}$ and $\sigma=\min \left\{\sigma_{i}(i=0,1,2, \ldots, m)\right\}$. In fact, for $r>0$, we have that $|x|,|y|,|z| \leq r$ imply that

$$
\left|f\left(t, t^{\alpha-4} x, t^{\alpha-4} y, t^{\alpha-4} z\right)\right| \leq t^{k}(1-t)^{\sigma}\left(M_{0}+\sum_{i=1}^{m} A_{i} r^{\tau_{i 1}} r^{\tau_{i 2}} r^{\tau_{i 3}}\right) .
$$

Then (B0) holds.

Let Banach space $X$, cone $P$ in $X$ and operator $T$ defined on $P$ be defined in Section 2. By Lemma 6, $T: P \rightarrow P$ is well defined, completely continuous, $x \in P$ is a positive solution if and only if $x \in P$ is a fixed point of $T$. Let

$$
\begin{aligned}
\Phi(t)= & \int_{0}^{t} \frac{(t-s)^{\alpha-1}}{\Gamma(\alpha)} \phi(s) d s+\left[(\alpha-2) t^{\alpha-1}-(\alpha-1) t^{\alpha-2}\right] \int_{0}^{1} \frac{(1-s)^{\alpha-1}}{\Gamma(\alpha)} \phi(s) d s \\
& +\left[t^{\alpha-2}-t^{\alpha-1}\right] \int_{0}^{1} \frac{(1-s)^{\alpha-2}}{\Gamma(\alpha-1)} \phi(s) d s .
\end{aligned}
$$

It is easy to see that $\Phi \in X$ and $\Phi(1)=\Phi^{\prime}(1)=0$. For $r>0$, denote $\Omega_{r}=\{x \in X \cap P:\|x-\Phi\| \leq r\}$. One sees that

$$
\|x\|=\max \left\{\begin{array}{l}
\sup _{t \in(0,1]} t^{4-\alpha}|x(t)|, \\
\sup _{t \in(0,1]} t^{4-\alpha}\left|x^{\prime}(t)\right|, \\
\sup _{t \in(0,1]} t^{4-\alpha}\left|x^{\prime \prime}(t)\right|
\end{array}\right\} \leq\|x-\Phi\|+\|\Phi\| \leq r+\|\Phi\|, \quad x \in \Omega_{r} .
$$


Hence for $x \in \Omega_{r}$, we have

$$
\begin{aligned}
& \left|f\left(t, x(t), x^{\prime}(t), x^{\prime \prime}(t)\right)-\phi(t)\right| \\
& \quad \leq \sum_{i=1}^{m} A_{i} t^{k_{i}}(1-t)^{\sigma_{i}}\left|t^{4-\alpha} x(t)\right|^{\tau_{i 1}}\left|t^{4-\alpha} x^{\prime}(t)\right|^{\tau_{i 2}}\left|t^{4-\alpha} x^{\prime \prime}(t)\right|^{\tau_{i 3}} \\
& \quad \leq \sum_{i=1}^{m} A_{i} t^{k_{i}}(1-t)^{\sigma_{i}}[r+\|\Phi\|]^{\mu_{i}} .
\end{aligned}
$$

Since for each $y \in X$ with $y(1)=y^{\prime}(1)=0$, there exist $\xi, \eta \in(t, 1]$ such that

$$
\begin{aligned}
t^{4-\alpha}|y(t)| & =t^{4-\alpha}|y(t)-y(1)|=t^{4-\alpha}\left|y^{\prime}(\xi)\right|(1-t) \\
& \leq \xi^{4-\alpha}\left|y^{\prime}(\xi)\right| \leq \sup _{t \in(0,1]} t^{4-\alpha}\left|y^{\prime}(t)\right|, \\
t^{4-\alpha}\left|y^{\prime}(t)\right| & =t^{4-\alpha}\left|y^{\prime}(t)-y^{\prime}(1)\right|=t^{4-\alpha}\left|y^{\prime \prime}(\eta)\right|(1-t) \\
& \leq \eta^{4-\alpha}\left|y^{\prime \prime}(\eta)\right| \leq \sup _{t \in(0,1]} t^{4-\alpha}\left|y^{\prime \prime}(t)\right| .
\end{aligned}
$$

We have

$$
\sup _{t \in(0,1]} t^{4-\alpha}|y(t)| \leq \sup _{t \in(0,1]} t^{4-\alpha}\left|y^{\prime}(t)\right| \leq \sup _{t \in(0,1]} t^{4-\alpha}\left|y^{\prime \prime}(t)\right|
$$

So

$$
\|y\|=\sup _{t \in(0,1]} t^{4-\alpha}\left|y^{\prime \prime}(t)\right|
$$

Then

$$
\begin{aligned}
t^{4-\alpha} \mid & (T x)^{\prime \prime}(t)-\Phi^{\prime \prime}(t) \mid \\
\leq & t^{4-\alpha} \int_{0}^{t} \frac{(t-s)^{\alpha-3}}{\Gamma(\alpha-2)}\left|f\left(s, x(s), x^{\prime}(s), x^{\prime \prime}(s)\right)-\phi(s)\right| d s \\
& +\left|(\alpha-2) t \frac{\Gamma(\alpha)}{\Gamma(\alpha-2)}-(\alpha-1) \frac{\Gamma(\alpha-1)}{\Gamma(\alpha-3)}\right| \\
& \times \int_{0}^{1} \frac{(1-s)^{\alpha-1}}{\Gamma(\alpha)}\left|f\left(s, x(s), x^{\prime}(s), x^{\prime \prime}(s)\right)-\phi(s)\right| d s \\
& +\left|\frac{\Gamma(\alpha-1)}{\Gamma(\alpha-3)}-t \frac{\Gamma(\alpha)}{\Gamma(\alpha-2)}\right| \\
& \times \int_{0}^{1} \frac{(1-s)^{\alpha-2}}{\Gamma(\alpha-1)}\left|f\left(s, x(s), x^{\prime}(s), x^{\prime \prime}(s)\right)-\phi(s)\right| d s \\
\leq & \sum_{i=1}^{m} A_{i}[r+\|\Phi\|]^{\mu_{i}} t^{2+k_{i}+\sigma_{i}} \int_{0}^{1} \frac{(1-w)^{\alpha+\sigma_{i}-3}}{\Gamma(\alpha-2)} w^{k_{i}} d w \\
& +\left[(\alpha-2) \frac{\Gamma(\alpha)}{\Gamma(\alpha-2)}+(\alpha-1) \frac{\Gamma(\alpha-1)}{\Gamma(\alpha-3)}\right] \\
& \times \sum_{i=1}^{m} A_{i} \frac{\mathbf{B}\left(\alpha+\sigma_{i}, k_{i}+1\right)}{\Gamma(\alpha)}[r+\|\Phi\|]^{\mu_{i}}
\end{aligned}
$$




$$
\begin{aligned}
& +\left[\frac{\Gamma(\alpha-1)}{\Gamma(\alpha-3)}+\frac{\Gamma(\alpha)}{\Gamma(\alpha-2)}\right] \sum_{i=1}^{m} A_{i} \frac{\mathbf{B}\left(\alpha+\sigma_{i}-1, k_{i}+1\right)}{\Gamma(\alpha-1)}[r+\|\Phi\|]^{\mu_{i}} \\
\leq & \sum_{i=1}^{m} A_{i} P_{i}[r+\|\Phi\|]^{\mu_{i}} .
\end{aligned}
$$

It follows the methods used in (3.1) that

$$
\sup _{t \in(0,1]} t^{4-\alpha}\left|(T x)^{\prime \prime}(t)-\Phi^{\prime \prime}(t)\right| \leq \sum_{i=1}^{m} A_{i} P_{i}[r+\|\Phi\|]^{\mu_{i}} .
$$

It is easy to show by the similar methods of (29) that

$$
\|T x-\Phi\|=\sup _{t \in(0,1]} t^{4-\alpha}\left|(T x)^{\prime \prime}(t)-\Phi^{\prime \prime}(t)\right| .
$$

Then (3.2) implies that

$$
\|T x-\Phi\| \leq P_{0} \sum_{i=1}^{m} A_{i} P_{i}[r+\|\Phi\|]^{\mu_{i}}
$$

Then

$$
\|T x-\Phi\| \leq[r+\|\Phi\|]^{\mu} \sum_{i=1}^{m} A_{i} P_{i}[r+\|\Phi\|]^{\mu_{i}-\mu} \leq \sum_{i=1}^{m} A_{i} P_{i}\|\Phi\|^{\mu_{i}-\mu}[r+\|\Phi\|]^{\mu} .
$$

Case 1. $\mu<1$.

Since there exists $r_{0}>0$ sufficiently large such that

$$
\sum_{i=1}^{m} A_{i} P_{i}\|\Phi\|^{\mu_{i}-\mu}\left[r_{0}+\|\Phi\|\right]^{\mu}<r_{0}
$$

Choose $\Omega_{r_{0}}=\left\{x \in X:\|x-\Phi\| \leq r_{0}\right\}$. From above discussion, we have

$$
\|T x-\Phi\| \leq \sum_{i=1}^{m} A_{i} P_{i}\|\Phi\|^{\mu_{i}-\mu}\left[r_{0}+\|\Phi\|\right]^{\mu} \leq r_{0} .
$$

Then $T x \in \Omega_{r_{0}}$. By Schauder fixed point theorem, $T$ has at least one fixed point $x \in \Omega_{r_{0}}$. Since $T: P \rightarrow P$, we know that $x$ is nonnegative on $(0,1]$. Then $x$ is a positive solution of BVP (1.7).

Case 2. $\mu=1$.

Choose $r_{0}>\sum_{i=1}^{m} A_{i} P_{i}\|\Phi\|^{\mu_{i}-\mu}\|\Phi\| /\left(1-\sum_{i=1}^{m} A_{i} P_{i}\|\Phi\|^{\mu_{i}-\mu}\right)$. Let $\Omega_{r_{0}}=$ $\left\{x \in X:\|x-\Phi\| \leq r_{0}\right\}$. From above discussion, we have

$$
\|T x-\Phi\| \leq \sum_{i=1}^{m} A_{i} P_{i}\|\Phi\|^{\mu_{i}-\mu}\left[r_{0}+\|\Phi\|\right] \leq r_{0} .
$$

Then $T x \in \Omega_{r_{0}}$. By Schauder fixed point theorem, $T$ has at least one fixed point $x \in \Omega$. Since $T: P \rightarrow P$, we know that $x$ is nonnegative on $(0,1]$. Then $x$ is a positive solution of BVP (1.7). 
Case 3. $\mu>1$.

Choose $r_{0}=\frac{\|\Phi\|}{\mu-1}$. Let $\Omega_{r_{0}}=\left\{x \in X:\|x-\Phi\| \leq r_{0}\right\}$. From above discussion, we have

$$
\|T x-\Phi\| \leq \sum_{i=1}^{m} A_{i} P_{i}\|\Phi\|^{\mu_{i}-\mu}\left[\frac{\|\Phi\|}{\mu-1}+\|\Phi\|\right]^{\mu} \leq \frac{\|\Phi\|}{\mu-1}=r_{0} .
$$

Then $T x \in \Omega_{r_{0}}$. By Schauder fixed point theorem, $T$ has at least one fixed point $x \in \Omega_{r_{0}}$. From $x \in P, T: P \rightarrow P$, we know that $x$ is nonnegative on $(0,1]$. Then $x$ is a positive solution of BVP (1.7).

The proof of Theorem 1 is completed.

Theorem 2. Suppose that (B0) holds. Furthermore, suppose that there exist positive constants $e_{1}, e_{2}, c$ with $0<e_{1}<e_{2}<e_{2} / \mu<c$. If

$$
\begin{aligned}
Q= & \frac{\mathbf{B}(\alpha+\sigma-2, k+1)}{\Gamma(\alpha-2)}+\left[(\alpha-2) \frac{\Gamma(\alpha)}{\Gamma(\alpha-2)}+(\alpha-1) \frac{\Gamma(\alpha-1)}{\Gamma(\alpha-3)}\right] \\
& \times \frac{\mathbf{B}(\alpha+\sigma, k+1)}{\Gamma(\alpha)}+\left[\frac{\Gamma(\alpha-1)}{\Gamma(\alpha-3)}+\frac{\Gamma(\alpha)}{\Gamma(\alpha-2)}\right] \frac{\mathbf{B}(\alpha+\sigma-1, k+1)}{\Gamma(\alpha-1)}, \\
W= & \frac{1}{e_{2}} \frac{(\alpha-2) \max \left\{p^{2}(1-p)^{2}, q^{2}(1-q)^{2}\right\}}{\Gamma(\alpha)} \int_{p}^{q} s^{2+k}(1-s)^{\alpha+\sigma-2} d s, \\
E= & \frac{1}{e_{1}}\left[2(\alpha-1)(\alpha-2) \frac{\mathbf{B}(\alpha+\sigma, k+1)}{\Gamma(\alpha)}+2(\alpha-1) \frac{\mathbf{B}(\alpha+\sigma-1, k+1)}{\Gamma(\alpha-1)}\right]
\end{aligned}
$$

with $Q<W$ and

(A1) $f\left(t, t^{\alpha-4} u, t^{\alpha-4} v, t^{\alpha-4} w\right)<\frac{t^{k}(1-t)^{\sigma}}{Q} c$ for all $t \in(0,1), u \in[0, c], v, w \in$ $[-c, c]$,

(A2) $f\left(t, t^{\alpha-4} u, t^{\alpha-4} v, t^{\alpha-4} w\right) \geq \frac{e_{2} t^{k}(1-t)^{\sigma}}{W}$ for all $t \in[p, q], u \in\left[e_{2}, \frac{e_{2}}{\mu}\right], v, w \in$ $[-c, c]$,

(A3) $f\left(t, t^{\alpha-4} u, t^{\alpha-4} v, t^{\alpha-4} w\right) \leq \frac{e_{1} t^{k}(1-t)^{\sigma}}{E}$ for all $t \in(0,1), u \in\left[0, e_{1}\right], v, w \in$ $[-c, c]$,

then BVP (1.7) has three positive solutions $x_{1}, x_{2}$ and $x_{3}$ such that

$$
\begin{array}{ll}
\sup _{t \in(0,1]} t^{4-\alpha} x_{1}(t)<e_{1}, & \min _{t \in[p, q]} t^{4-\alpha} x_{2}(t)>e_{2}, \\
\sup _{t \in(0,1]} t^{4-\alpha} x_{3}(t)>e_{1}, & \min _{t \in[p, q]} t^{4-\alpha} x_{3}(t)<e_{2} .
\end{array}
$$

Proof. Let Banach space $X$, cone $P$ in $X$ and operator $T$ defined on $P$ be defined in Section 2. By Lemma 6, $T: P \rightarrow P$ is well defined, completely continuous, $x \in P$ is a positive solution if and only if $x \in P$ is a fixed point of $T$.

Let $\mu$ be defined in Lemma 5. Define the functionals by

$$
\beta_{1}(y)=\sup _{t \in(0,1]} t^{4-\alpha}\left|y^{\prime \prime}(t)\right|, \quad y \in P, \quad \beta_{2}(y)=\sup _{t \in(0,1]} t^{4-\alpha}|y(t)|, \quad y \in P,
$$




$$
\begin{aligned}
& \beta_{3}(y)=\sup _{t \in(0,1]} t^{4-\alpha}|y(t)|, \quad y \in P, \quad \alpha_{1}(y)=\min _{t \in[p, q]} t^{4-\alpha}|y(t)|, \quad y \in P, \\
& \alpha_{2}(y)=\min _{t \in[p, q]} t^{4-\alpha}|y(t)|, \quad y \in P .
\end{aligned}
$$

It is easy to see that $\alpha_{1}, \alpha_{2}$ are two nonnegative continuous concave functionals on the cone $P, \beta_{1}, \beta_{2}, \beta_{3}$ are three nonnegative continuous convex functionals on the cone $P$.

It follows that $\alpha_{1}(x) \leq \beta_{2}(x)$ for all $x \in P$. We can show by similar methods of (29) that

$$
\|x\|=\sup _{t \in(0,1]} t^{4-\alpha}\left|x^{\prime \prime}(t)\right| \leq \beta_{1}(x) \quad \text { for all } x \in P .
$$

From above discussion, (i) and (ii) in Lemma 1 hold.

Now, we prove that (iii) in Lemma 1 holds. Choose $c_{5}=c, c_{4}=\mu e_{1}$, $c_{3}=e_{2} / \mu, c_{2}=e_{2}, c_{1}=e_{1}$. One sees that $0<c_{1}<c_{2}$. The remainder is divided into five steps.

Step 1. Prove that $T: \overline{P_{c_{5}}} \rightarrow \overline{P_{c_{5}}}$;

For $u \in \overline{P_{c}}$, we have $\|u\| \leq c$. Then $0 \leq t^{4-\alpha} u(t) \leq c,-c \leq t^{4-\alpha} u^{\prime}(t) \leq c$ and $-c \leq t^{4-\alpha} u^{\prime \prime}(t) \leq c$ for all $t \in(0,1]$. So (A1) implies that

$$
f\left(t, u(t), u^{\prime}(t), u^{\prime \prime}(t)\right) \leq \frac{c t^{k}(1-t)^{\sigma}}{Q}, \quad t \in(0,1) .
$$

By the definition of $T$, we have

$$
\begin{aligned}
& t^{4-\alpha}\left|(T u)^{\prime \prime}(t)\right| \leq \frac{\mathbf{B}(\alpha+\sigma-2, k+1)}{\Gamma(\alpha-2)} \frac{c}{Q} \\
& +\left[(\alpha-2) \frac{\Gamma(\alpha)}{\Gamma(\alpha-2)}+(\alpha-1) \frac{\Gamma(\alpha-1)}{\Gamma(\alpha-3)}\right] \frac{\mathbf{B}(\alpha+\sigma, k+1)}{\Gamma(\alpha)} \frac{c}{Q} \\
& +\left[\frac{\Gamma(\alpha-1)}{\Gamma(\alpha-3)}+\frac{\Gamma(\alpha)}{\Gamma(\alpha-2)}\right] \frac{\mathbf{B}(\alpha+\sigma-1, k+1)}{\Gamma(\alpha-1)} \frac{c}{Q} \leq c .
\end{aligned}
$$

By definition of $T u$, we get from similar methods of (3.1) that

$$
\sup _{t \in(0,1]} t^{4-\alpha}|(T u)(t)| \leq \sup _{t \in(0,1]} t^{4-\alpha}\left|(T u)^{\prime}(t)\right| \leq \sup _{t \in(0,1]} t^{4-\alpha}\left|(T u)^{\prime \prime}(t)\right| .
$$

It follows that $\|T u\| \leq c$. Then $T u \in \overline{P_{c}}$. Then $T: \overline{P_{c}} \rightarrow \overline{P_{c}}$. Hence $(3)-(\mathrm{C} 1)$ holds.

Step 2. Prove that $\alpha_{1}(T y)>c_{2}$ for $y \in P\left(\beta_{1}, \alpha_{1} ; c_{2}, c_{5}\right)$ with $\beta_{3}(T y)>c_{3}$; For $y \in P\left(\beta_{1}, \alpha_{1} ; a_{2}, a_{5}\right)=P\left(\beta_{1}, \alpha_{1} ; e_{2}, c\right)$ with $\beta_{3}(T y)>\frac{e_{2}}{\mu}$, we have that $\alpha_{1}(y) \geq e_{2}, \beta_{1}(y) \leq c$, and

$$
\sup _{t \in(0,1]} t^{4-\alpha}(T y)(t)>\frac{e_{2}}{\mu}
$$

Hence $T y \in P$ implies that

$$
\alpha_{1}(T y)=\min _{t \in[p, q]}(T y)(t) \geq \mu \sup _{t \in(0,1]} t^{4-\alpha}(T y)(t)>\mu \frac{e_{2}}{\mu}=e_{2}=c_{2}
$$


This completes the proof of $(3)-(\mathrm{C} 2)$.

Step 3. Prove that $\beta_{2}(T y)<c_{1}$ for each $y \in Q\left(\beta_{1}, \beta_{2} ; c_{1}, c_{5}\right)$ with $\alpha_{2}(T y)<c_{4}$.

For $y \in Q\left(\beta_{1}, \beta_{2} ; c_{1}, c_{5}\right)$ with $\alpha_{2}(T y)<c_{4}$, we have that $\beta_{1}(y) \leq c_{5}=c$, $\beta_{2}(y) \leq c_{1}=e_{1}$, and $\alpha_{2}(T y)<c_{4}=\mu e_{1}$. Then

$$
\beta_{2}(T y)=\sup _{t \in(0,1]} t^{4-\alpha}(T y)(t) \leq \frac{1}{\mu} \min _{t \in[p, q]} t^{4-\alpha}(T y)(t)<\frac{1}{\mu} \mu e_{1}=c_{1} .
$$

This completes the proof of $(3)-(\mathrm{C} 3)$.

Step 4. Prove that $\left\{y \in P\left(\beta_{1}, \beta_{3}, \alpha_{1} ; c_{2}, c_{3}, c_{5}\right): \alpha_{1}(x)>c_{2}\right\} \neq \emptyset$ and $\alpha_{1}(T y)>e_{2}$ for every $y \in P\left(\beta_{1}, \beta_{3}, \alpha_{1} ; e_{2}, \frac{e_{2}}{\mu}, c_{1}\right)$;

It is easy to see that $\left\{y \in P\left(\beta_{1}, \beta_{3}, \alpha_{1} ; e_{2}, \frac{e_{2}}{\mu}, c_{1}\right): \alpha_{1}(y)>e_{2}\right\} \neq \emptyset$.

For $y \in P\left(\beta_{1}, \beta_{3}, \alpha_{1} ; e_{2}, \frac{e_{2}}{\mu}, c_{1}\right)$, one has that $\alpha_{1}(y) \geq e_{2}, \beta_{3}(y) \leq \frac{e_{2}}{\mu}$, $\beta_{1}(y) \leq c_{1}$. Then

$$
e_{2} \leq t^{4-\alpha} y(t) \leq \frac{e_{2}}{\mu}, \quad t \in[p, q], \quad t^{4-\alpha}\left|y^{\prime}(t)\right| \leq c_{5}, t^{4-\alpha}\left|y^{\prime \prime}(t)\right| \leq c_{5} .
$$

Thus (A2) implies that

$$
f\left(t, y(t), y^{\prime}(t), y^{\prime \prime}(t)\right) \geq \frac{e_{2} t^{k}(1-t)^{\sigma}}{W}, \quad t \in[p, q] .
$$

For $t \in[p, q],(7)$ implies that

$$
\begin{aligned}
t^{4-\alpha} & (T y)(t) \geq \int_{p}^{q} \frac{(\alpha-2) t^{2}(1-t)^{2} s^{2}(1-s)^{\alpha-2}}{\Gamma(\alpha)} \frac{e_{2} s^{k}(1-s)^{\sigma}}{W} d s \\
& \geq \frac{(\alpha-2) \max \left\{p^{2}(1-p)^{2}, q^{2}(1-q)^{2}\right\}}{\Gamma(\alpha)} \int_{k}^{l} s^{2+k}(1-s)^{\alpha+\sigma-2} d s \frac{e_{2}}{W}=e_{2} .
\end{aligned}
$$

This completes the proof of (3)-(C4).

Step 5. Prove that $\left\{y \in Q\left(\beta_{1}, \beta_{3}, \alpha_{2} ; c_{4}, c_{1}, c_{5}\right): \beta_{2}(x)<c_{1}\right\} \neq \emptyset$ and $\beta_{2}(T x)<c_{1}$ for every $x \in Q\left(\beta_{1}, \beta_{3}, \alpha_{2} ; c_{4}, c_{1}, c_{5}\right)$;

It is easy to see that $\left\{y \in Q\left(\beta_{1}, \beta_{3}, \alpha_{2} ; c_{4}, c_{1}, c_{5}\right): \beta_{2}(x)<c_{1}\right\} \neq \emptyset$. For $y \in Q\left(\beta_{1}, \beta_{3}, \alpha_{2} ; c_{4}, c_{1}, c_{5}\right)$, one has that $\alpha_{2}(y) \geq c_{4}=\mu e_{1}, \beta_{3}(y) \leq c_{1}=e_{1}$, $\beta_{1}(y) \leq c_{5}=c$. Hence we get that

$$
\begin{aligned}
0 & \leq t^{4-\alpha} y(t) \leq e_{1}, \quad t \in[0,1] \\
-c & \leq t^{4-\alpha} y^{\prime}(t) \leq c, \quad-c \leq t^{4-\alpha} y^{\prime \prime}(t) \leq c, \quad t \in(0,1) .
\end{aligned}
$$

Then (A3) implies that

$$
f\left(t, y(t), y^{\prime}(t), y^{\prime \prime}(t)\right) \leq e_{1} t^{k}(1-t)^{\sigma} / E, \quad t \in(0,1) .
$$

Similarly to Step 1, we get that

$$
\beta_{2}(T y)=\sup _{t \in(0,1]} t^{4-\alpha}(T y)(t) \leq \sup _{t \in(0,1]} t^{4-\alpha}(T y)^{\prime \prime}(t) \leq e_{1} .
$$

This completes the proof of (3)-(C5).

Then Lemma 1 implies that $T$ has at least three fixed points $y_{1}, y_{2}$ and $y_{3}$ such that $\beta_{2}\left(y_{1}\right)<e_{1}, \alpha_{1}\left(y_{2}\right)>e_{2}, \beta_{2}\left(y_{3}\right)>e_{1}, \alpha_{1}\left(y_{3}\right)<e_{2}$. Hence BVP (1.7) has three positive solutions $x_{1}, x_{2}$ and $x_{3}$ such that (3.3) holds. The proof is complete. 


\section{Acknowledgments}

The author would like to thank the referees and editors for their careful reading and some comments on improving the presentation of this paper.

\section{References}

[1] R.P. Agarwal and Y.M. Chow. Iterative method for fourth order boundary value problem. J. Comput. Appl. Math., 10:203-217, 1984. http://dx.doi.org/10.1016/0377-0427(84)90058-X.

[2] A. Arara, M. Benchohra, N. Hamidi and J.J. Nieto. Fractional order differential equations on an unbounded domain. Nonlinear Anal., 72:580-586, 2010. http://dx.doi.org/10.1016/j.na.2009.06.106.

[3] T.M. Atanackovic and B. Stankovic. On a system of differential equations with fractional derivatives arising in rod theory. J. Phys. A, 37:1241-1250, 2004. http://dx.doi.org/10.1088/0305-4470/37/4/012.

[4] R.I. Avery. A generalization of the Leggett-Williams fixed-point theorem. Math. Sci. Res. Hot-line, 2:9-14, 1998.

[5] C. Bai. Triple positive solutions for a boundary value problem of nonlinear fractional differential equation. Electron. J. Qual. Theory Differ. Equ., 24:1-10, 2008 .

[6] Z. Bai. The upper and lower solution method for some fourth-order boundary value problems. Nonlinear Anal., 67:1704-1709, 2007.

http://dx.doi.org/10.1016/j.na.2006.08.009.

[7] Z. Bai. On positive solutions of a nonlocal fractional boundary value problem. Nonlinear Anal., 72:916-924, 2010.

http://dx.doi.org/10.1016/j.na.2009.07.033.

[8] Z. Cui, P. Yu and Z. Mao. Existence of solutions for nonlocal boundary value problems of nonlinear fractional differential equations. Adv. Dyn. Syst. Appl., 7:31-40, 2012.

[9] R. Dehghant and K. Ghanbari. Triple positive solutions for boundary value problem of a nonlinear fractional differential equation. Bull. Iran. Math. Soc., 33:1-14, 2007.

[10] A. Dzielinski and D. Sierociuk. Fractional order model of beam heating process and its experimental verification. In D. Baleanu, Z.B. Guvenc and J.A. Tenreiro Machado(Eds.), New Trends in Nanotechnology and Fractional Calculus Applications, Dordrecht, Heidelberg, London, Newyrk, 2010. Springer.

[11] A.M.A. El-Sayed, A.E.M. El-Mesiry and H.A.A. El-Saka. On the fractional order logistic equation. Appl. Math. Lett., 20:817-823, 2007.

http://dx.doi.org/10.1016/j.aml.2006.08.013.

[12] J.H. He. Nonlinear oscillation with fractional derivative and its applications. In International Conference on Vibration Engineering '98, pp. 288-291, Dalian, China, 1998.

[13] A.A. Kilbas, H.M. Srivastava and J.J. Trujillo. Theory and Applications of Fractional Differential Equations, volume 204 of North-Holland Math. Stud. Elsevier Science, Amsterdam, The Netherlands, 2006. 
[14] A.A. Kilbas and J.J. Trujillo. Computation of fractional integrals via functions of hypergeometric and Bessel type. J. Comput. Appl. Math., 118:223-239, 2000. http://dx.doi.org/10.1016/S0377-0427(00)00291-0.

[15] A.A. Kilbas and J.J. Trujillo. Differential equations of fractional order: methods, results and problems-I. Appl. Anal., 78:153-192, 2001.

http://dx.doi.org/10.1080/00036810108840931.

[16] V. Lakshmikantham and A.S. Vatsala. Basic theory of fractional differential equations. Nonlinear Anal., 69:2677-2682, 2008.

http://dx.doi.org/10.1016/j.na.2007.08.042.

[17] S. Li and X. Zhang. Existence and uniqueness of monotone positive solutions for an elastic beam equation with nonlinear boundary conditions. Comput. Math. Appl., 63:1355-1360, 2012. http://dx.doi.org/10.1016/j.camwa.2011.12.065.

[18] S. Liang and J. Zhang. Positive solutions for boundary value problems of nonlinear fractional differential equations. Nonlinear Anal., 71:5545-5550, 2009. http://dx.doi.org/10.1016/j.na.2009.04.045.

[19] H. Lu, L. Sun and J. Sun. Existence of positive solutions to a non-positive elastic beam equation with both ends fixed. Bound. Value Probl., 2012(56):1-10, 2012.

[20] R. Ma. Existence of positive solutions of a fourth order boundary value problem. Appl. Math. Comput., 168:1219-1231, 2005. http://dx.doi.org/10.1016/j.amc.2004.10.014.

[21] K.S. Miller and B. Ross. An introduction to the Fractional Calculus and Fractional Differential Equation. Wiley, New York, 1993.

[22] S.Z. Rida, H.M. El-Sherbiny and A.A.M. Arafa. On the solution of the fractional nonlinear Schrödinger equation. Phys. Lett. A, 372:553-558, 2008. http://dx.doi.org/10.1016/j.physleta.2007.06.071.

[23] S.G. Samko, A.A. Kilbas and O.I. Marichev. Fractional Integral and Derivative, Theory and Applications. Gordon and Breach, 1993.

[24] X. Xu, D. Jiang and C. Yuan. Multiple positive solutions for the boundary value problem of a nonlinear fractional differential equation. Nonlinear Anal., 71:4676-4688, 2009. http://dx.doi.org/10.1016/j.na.2009.03.030.

[25] Q. Yao. Positive solutions for eigenvalue problems of four-order elastic beam equations. Appl. Math. Lett., 17:237-243, 2004. http://dx.doi.org/10.1016/S0893-9659(04)90037-7.

[26] F. Zhang. Existence results of positive solutions to boundary value problem for fractional differential equation. Positivity, 13:583-599, 2008. http://dx.doi.org/10.1007/s11117-008-2260-5.

[27] S.Q. Zhang. Monotone iterative method for initial value problem involving Riemann-Liouville fractional derivatives. Nonlinear Anal., 71:2087-2093, 2009. http://dx.doi.org/10.1016/j.na.2009.01.043. 